\title{
Surgery combined with adenoviral p53 gene therapy for treatment of non-small cell lung cancer: a phase II study
}

\author{
Bo Deng ${ }^{1, *}$, Tianyu Sun ${ }^{1, *}$, Bo Tang ${ }^{1, *}$, Shaolin Tao ${ }^{1}$, Poming Kang ${ }^{1}$, Kai Qian ${ }^{1}$, Bin \\ Jiang ${ }^{1}$, Kun Li ${ }^{1}$, Kunkun Li ${ }^{1}$, Jinghai Zhou ${ }^{1}$, Ruwen Wang ${ }^{1}$ and Qunyou Tan ${ }^{1}$ \\ ${ }^{1}$ Department of Thoracic Surgery, Institute of Surgery Research, Daping Hospital, Third Military Medical University, Chongqing \\ 400042, China \\ *These authors have contributed equally to this work \\ Correspondence to: Qunyou Tan, email: tanqy001@163.com \\ Keywords: adenoviral p53 gene; non-small cell lung cancer; radical surgery; recurrence; metastasis \\ Received: June 27, $2017 \quad$ Accepted: September 20,2017 Published: November 06, 2017 \\ Copyright: Deng et al. This is an open-access article distributed under the terms of the Creative Commons Attribution License 3.0 \\ (CC BY 3.0), which permits unrestricted use, distribution, and reproduction in any medium, provided the original author and source \\ are credited.
}

\section{ABSTRACT}

Objective: To assess the efficacy of radical surgery combined with recombinant adenoviral human p53 (rAd-p53) gene therapy in treatment of resectable non-small cell lung cancer.

Method: A total of 163 patients with resectable NSCLC meeting the inclusion criteria were randomly assigned to two groups: radical surgery alone (S) and radical surgery plus surgical wound surface injection of $2 \times 10^{12}$ rAd-p53 units (SP). All patients were followed up for at least 3 years for efficacy and safety. Study endpoints were loco-regional recurrence or distant metastasis (Rec-Met) rate as primary endpoints, and progression free survival (PFS), overall survival (OS) and safety assessments as secondary endpoints.

Results: Recurrence or metastasis (Rec/Met) after surgery were 24/82 $(29.27 \%)$ in SP group and $37 / 81(45.68 \%)$ in S group. The difference in the Rec/Met rate was statistically significant $(p=0.0304)$ by chi-square test. The hazard ratios after adjusting of age and disease stage (S vs. SP) of PFS and OS are 1.772 (95\% CI, 1.102 to 2.848 ) and 2.047 (95\% CI, 1.109 to 3.377), respectively. The 3 years PFS and OS for SP vs. S were $71.9 \%$ vs. $46.9 \%$, and $88.4 \%$ vs. $67.0 \%$, respectively. Differences in PFS and OS between two treatment groups were significant with the $p$ values of 0.0165 and 0.0191 , respectively, using Log-Rank test.

Conclusions: The wound surface injection of rAd-p53 showed efficacious effects in preventing recurrence or metastasis and improving PFS and OS after a radical surgery in patients with NSCLC.

\section{INTRODUCTION}

In the past 30 years, the incidence and mortality of lung cancer significantly increased. Lung cancer is the leading cause of death for all kinds of cancer in men and women worldwide. The American Cancer Society's estimates for lung cancer in the United States for 2016 are: about 224,390 new cases of lung cancer $(117,920$ in men and 106,470 in women) and about158,080 deaths from lung cancer (85,920 in men and 72,160 in women). In China, over 1.6 million of the new cases are diagnosed each year, accounting for $12.7 \%$ of new cases for all cancer types, and over 1.37 million of the lung cancer patients die each year. The non-small cell lung cancer (NSCLC) is the most common type of lung cancer and accounts for about $75 \%-85 \%$ of all cases.

Radical surgery still remains the main and potentially curative treatment for the early stage NSCLC. 
The five-year survival rate is from $30 \%$ to $70 \%$ after a radical surgery depending on the disease stage [1-3]. Local recurrence or remote metastasis is the main cause of death after a radical surgery. It is estimated $30 \%$ to $80 \%$ of patients with NSCLC will develop local recurrence or remote metastasis in 5 years after surgery $[4,5]$. Therefore, there is a need to further improve the NSCLC patients' survival by preventing or delaying the recurrence or metastasis after radical surgery.

P53 gene is one of the most important tumor suppressing genes. It has multiple anti-tumor functions including blocking of the cell cycle, inducing cell apoptosis, inhibiting tumor angiogenesis and sensitizing tumor to chemo- and radio-therapy [6-8]. P53 mutations are common in NSCLC with occurrence of $70 \%$ [9]. Loss of p53 normal functions is associated with tumorigenesis, and chemo- and radio-therapy resistance [10]. Gendicine ${ }^{\circledR}$, an antitumor gene product approved for marketing by the Chinese Food and Drug Administration (CFDA) in 2004, is a recombinant adenoviral p53 gene (rAd-p53), in which a wild type p53 CDNA was inserted in a replication-defective adenovirus type 5. A phase II study indicated that intratumoral injection of rAd-p53 can improve 5-years overall survival (OS) and progress free survival (PFS) when combined with radiotherapy in treatment of patients with advanced head and neck cancer [11]. It also has been shown that injection of rAd-p53 to the surgical wound could prevent or delay the recurrence of oral cancer after radical surgery [12]. Common adverse events associated with using rAd-p53 are self-limiting mild to moderate fever $[11,12]$. So far, no serious adverse effect related to rAd-p53 has been reported.

The objectives of this study are to investigate the effects of surgical wound surface injection of rAd-p53 to prevent recurrence or metastasis after radical surgery for patients with NSCLC.

\section{RESULTS}

\section{Patients and disease characteristics}

From March 10 of 2012 to June 30 of 2013, a total of 180 of patients recruited, and 163 of the patients met the inclusion criteria and were randomly assigned to either one of the two treatment groups: 82 patients in the SP group and 81 in the $\mathrm{S}$ group. Patients' demographics and baseline disease characteristic were summarized in Table 1 and Table 2 , respectively.

\section{Surgery}

For all the patients, the visible tumors were successfully removed by one of the surgical procedures: lobectomy, segmentectomy, or pneumonectomy. The detailed summary of the surgical procedures are showed in Table 3.

\section{Loco-regional recurrence and distant metastasis}

The loco-regional recurrences (Rec) are the recurrent tumors after surgery, which located within the same hemi-thorax as the original tumor including ipsilateral lung, lymph nodes, the bronchial stump, and the pleura and chest wall. The recurrent tumors located remotely from the local region are considered as distant metastases (Met). At 3 years after surgery, there were 24/82 (29.27\%) patients in SP group developing either Rec or Met (Rec/Met), compared with 37/81 (45.68\%) patients in S group. There were 3 patients who had both Rec and Met in SP group and 8 patients who had both Rec and Met in S group, which could explain that the number of Rec and Met in SP group was 24 instead of 27 and the number of Rec and Met in S group was 37 instead of 45 . The difference in Rec/Met rate is statistically significant ( $p=0.0304)$ using chi-square test. The Rec/Met data was summarized in Table 4.

\section{PFS and OS}

At the time of this analysis, the minimal follow-up time was 3 years and the median follow-up was 3.6 years with a range of 3 to 4.8 years. The number of patients with an outcome of death (17) or Rec/Met (12) was 29 for the SP group, compared with a total 42 of deaths (26) or (14) in the S group. The 3-years PFS and OS for SP vs. S are $71.9 \%$ vs. $46.9 \%$, and $88.4 \%$ vs. $67.0 \%$, respectively.

The difference in PFS between the two groups is statistically significant ( $\mathrm{P}=0.0165$ ) using log-rank test. The median PFS of SP group was 46 months with $95 \%$ CI of 38.2 months to 53.5 months, comparing to the $\mathrm{S}$ group with median PFS of 34 months with $95 \%$ CI of 31.8 to 41.9 months. The hazard ratio of $\mathrm{S}$ vs. SP group for PFS is 1.772 with $95 \%$ CI of 1.102 to 2.848 using Cox model adjusted by age and disease stage. The PFS plots are shown in the Figure 1.

The difference in OS between the two groups is statistically significant ( $\mathrm{P}=0.0191)$ using log-rank test. The median OS of SP group was 50.8 months with 95\% CI of 45.8 months to 56.3 months, comparing to $\mathrm{S}$ group with median OS of 44.6 months with $95 \%$ CI of 36.8 months to 51.7 months. The hazard ratio of S vs. SP group is 2.047 with $95 \%$ CI of 1.109 to 3.377 adjusted by age and disease stage. The OS plots are shown in the Figure 2.

\section{Adverse events and complications}

The adverse events and surgical complications were summarized in Table 5. Among them, only fever was classed as the rAd-p53 related adverse event. 


\begin{tabular}{llr} 
Age (years) & & \\
Mean (SD) & $58.4(12.9)$ & $57.1(11.3)$ \\
Median (Min, Max) & $61(39-75)$ & $59(41-73)$ \\
Sex & & $54(66.7 \%)$ \\
Male & $57(65.5 \%)$ & $27(33.3 \%)$ \\
Female & $25(34.5 \%)$ & $45(55.6 \%)$ \\
Smoking History & & $36(44.4 \%)$ \\
Yes & $48(58.5 \%)$ & $34(41.5 \%)$ \\
No & & \\
\hline
\end{tabular}

Table 2: Disease Characteristics at Baseline

\begin{tabular}{|c|c|c|}
\hline & Surgery plus rAd-p53 N=82 n (\%) & Surgery alone $\mathrm{N}=81 \mathrm{n}(\%)$ \\
\hline \multicolumn{3}{|l|}{ Histology Type } \\
\hline Adenocarcinoma & $37(45.1)$ & $32(39.5)$ \\
\hline Squamous & $32(39.0)$ & $25(30.9)$ \\
\hline Large Cell & $5(6.1)$ & $8(9.9)$ \\
\hline Undifferentiated & $1(1.2)$ & $2(2.5)$ \\
\hline Other & $7(8.5)$ & $14(17.3)$ \\
\hline \multicolumn{3}{|l|}{ Stage } \\
\hline I & $33(40.2)$ & $36(44.4)$ \\
\hline II & $37(45.1)$ & $34(42.0)$ \\
\hline IIIa & $12(14.6 \%)$ & $11(13.6)$ \\
\hline \multicolumn{3}{|l|}{ TNM stage } \\
\hline Ia (T1N0) & $15(18.3)$ & $14(17.3)$ \\
\hline Ib (T2N0) & $18(22.0)$ & $22(27.2)$ \\
\hline IIa (T1N1) & $19(23.2)$ & $17(21.0)$ \\
\hline $\mathrm{IIb}(\mathrm{T} 2 \mathrm{~N} 1, \mathrm{~T} 3 \mathrm{~N} 0)$ & $18(22.0)$ & $17(21.0)$ \\
\hline IIIa & $12(14.6)$ & $11(13.6)$ \\
\hline \multicolumn{3}{|l|}{$\mathrm{ECOG}$} \\
\hline 0 & $47(57.3)$ & $48(59.3)$ \\
\hline 1 & $35(42.7)$ & $33(40.7)$ \\
\hline
\end{tabular}

\section{DISCUSSION}

Local recurrence and remote metastasis are still the main cause of death after a radical surgery for NSCLC. Decrease in the Rec/Met rates will further improve the patients' survival. One may immediately think of the method to reduce the rate was to choose an appropriate surgical procedure and completely remove the residual tumor cells according to accurate assessment of the extent of tumor invasion based on imaging scans and observation during surgery. But this method is mostly dependent on a surgeon's experience and also tumor size and location.

A number of studies indicated that both pre- and post-surgery neoadjuvant and adjuvant can improve patients' PFS and OS [13-16]. One randomized phase III study compared between surgery alone and surgery plus 
Table 3: Surgical Procedures

\begin{tabular}{lcc}
\hline Surgery & Surgery plus rAd-p53 N=82 n (\%) & Surgery alone N=81 n $(\%)$ \\
\hline Lobectomy & $63(76.8)$ & $58(71.6)$ \\
Segmentectomy & $11(13.4)$ & $10(12.3)$ \\
Pneumonectomy & $8(9.8)$ & $13(16.1)$ \\
\hline
\end{tabular}

Table 4: Loco-regional Recurrence and Distant Metastasis

\begin{tabular}{lcc}
\hline Rec/Met & Surgery plus rAd-p53 N=82 n (\%) & Surgery alone N=81 n (\%) \\
\hline Any Rec & $9(11.0)$ & $20(24.7)$ \\
Lung Around Surgical Margin & $5(6.1)$ & $13(16.0)$ \\
Lymph Nodes & $6(7.3)$ & $9(11.1)$ \\
Pleura & $4(4.9)$ & $7(8.6)$ \\
Chest wall & $1(1.2)$ & $3(3.7)$ \\
Any Met & $18(22.0)$ & $25(30.7)$ \\
Brain & $8(9.8)$ & $11(13.6)$ \\
Bone & $6(7.3)$ & $9(11.1)$ \\
Liver & $5(6.1)$ & $8(9.9)$ \\
Adrenal & $3(3.7)$ & $5(6.2)$ \\
Rec and Met & $24(29.3)$ & $37(45.7)$ \\
\hline
\end{tabular}

Table 5: Adverse Events and Complications

\begin{tabular}{lcc}
\hline & Surgery plus rAd-p53 N=82 n (\%) & Surgery alone N=81 n $(\%)$ \\
\hline Pneumonia & $29(35.4)$ & $22(27.2)$ \\
Respiratory failure & $13(15.9)$ & $8(9.8)$ \\
Hemorrhage & 0 & $2(2.5)$ \\
Bronchial fistula & 0 & $1(1.2)$ \\
Arrhythmia & $5(6.1)$ & $4(4.9)$ \\
Recurrent laryngeal nerve injury & $1(1.2)$ & 0 \\
Fever $\left(>38^{\circ} \mathrm{C}\right)$ & $49(59.8)$ & $6(7.4)$
\end{tabular}

pre-operative cisplatin and gemcitabine for treatment of stage IB to IIIA NSCLC [17]. The hazard ratios for PFS and overall survival were 0.70 (95\% CI, 0.50 to 0.97$)$ and 0.63 (95\% CI, 0.43 to 0.92 ), respectively, both in favor of chemotherapy plus surgery. In this report the authors reviewed several similar studies and all these studies showed pre-operative chemotherapy could improve the NSCLC patients' survival. Suehisa H and Toyooka S reviewed the post-surgery adjuvant chemotherapy for completely resected non-small-cell lung cancer [18]. In this review, several studies showed the post-surgery adjuvant chemotherapy improved the 5-year survival from $4.1 \%$ to $11 \%$. In this study, neither pre- nor post-operative chemotherapy was used because of the concern that serious adverse events from pre-operative chemotherapy might delay surgery, and that post-operative chemotherapy might be ineffective and could cause serious adverse effects. However, a long term follow up is needed to fully define the beneficial effects of rAd-p53.

Considering the multiple anti-tumor functions of p53 gene and the favorable safety profile of rAd-p53 we used rAd-p53 as an adjuvant therapy for completely 


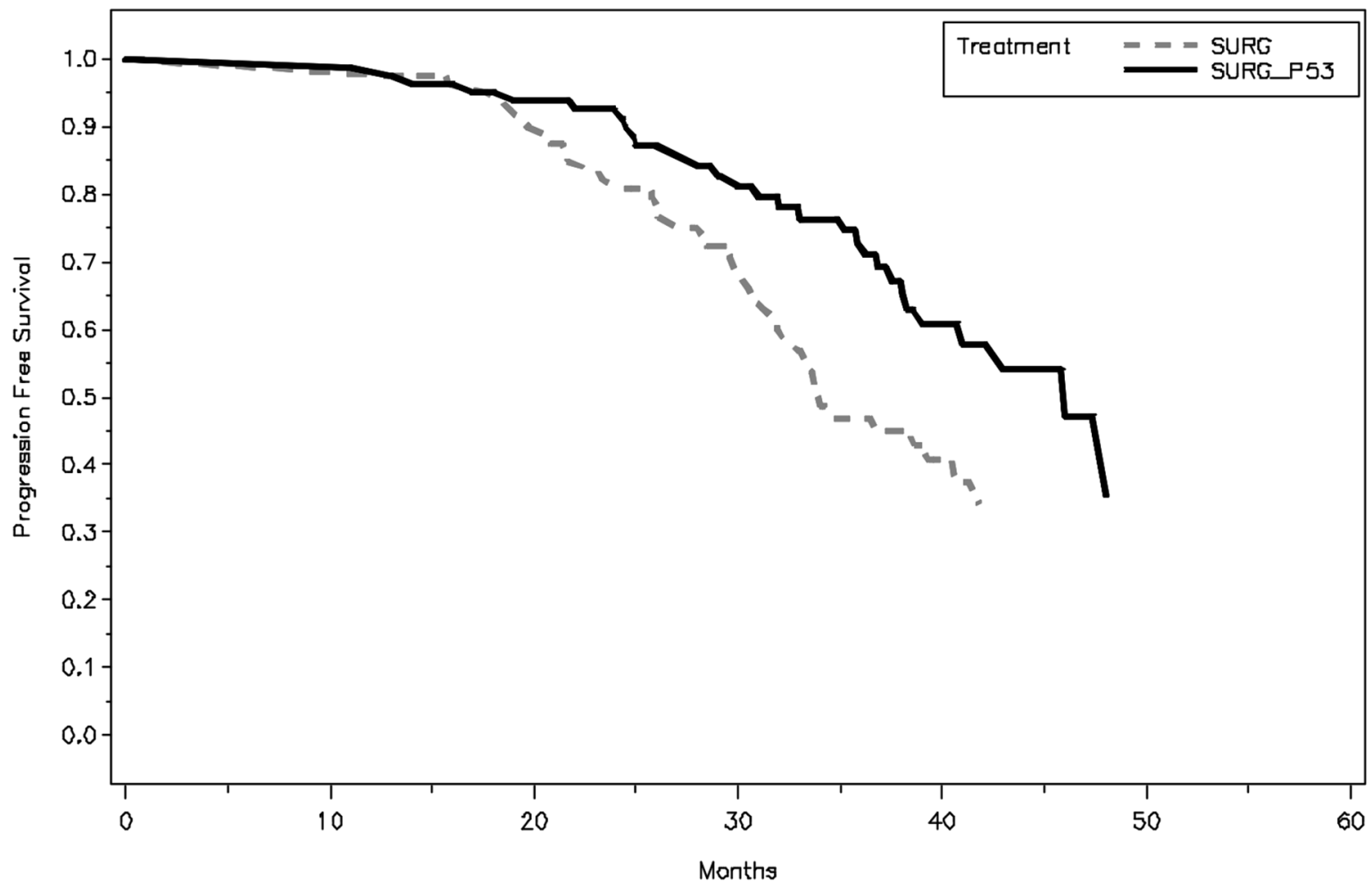

Figure 1: Kaplan-Meier plot of progression-free survival (PFS), assessed from surgery to the time when the last patient finished his 3-years follow-up.

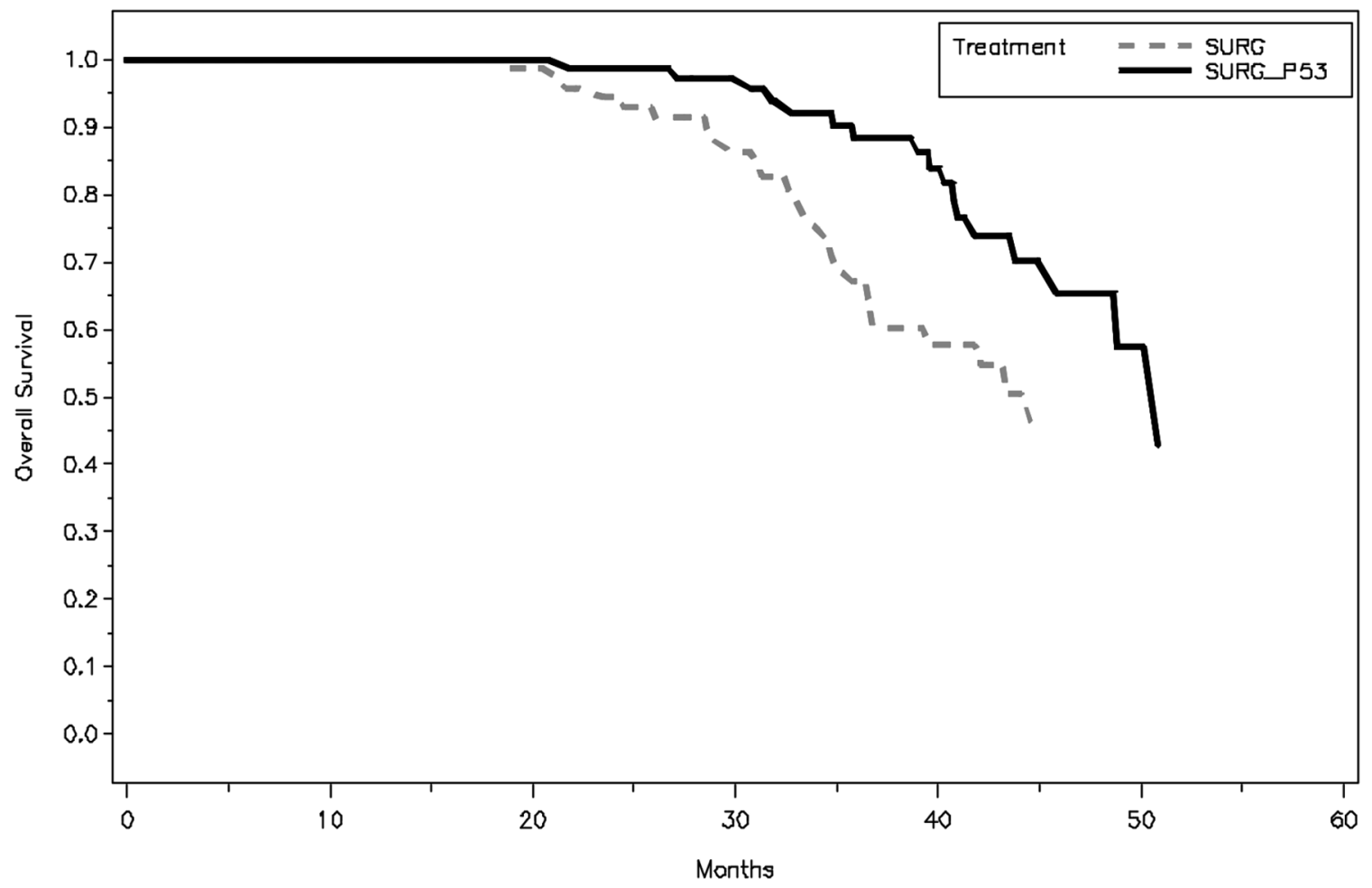

Figure 2: Kaplan-Meier plot of overall survival (OS), assessed from surgery to the time when the last patient finished his 3-years follow-up and his follow-up was the shortest. 
resected NSCLC. The results showed that rAd-p53 significantly lowered the Rec/Met rate $(p=0.0304)$ and improved both PFS and OS.

The rAd-p53 related adverse event was selflimited fever. The Fever $>38^{\circ} \mathrm{C}$ was observed in $59.8 \%$ of the SP group versus $7.4 \%$ of S group. The fever was from $38^{\circ} \mathrm{C}$ to $40.6^{\circ} \mathrm{C}$, occurred 2 to 6 days after the surgery and usually lasted 2-5 days. To most of the patients, physical methods were effective in relieving the fever. No other rAd-p53 related adverse event was observed.

In conclusion, the wound surface injection of rAd-p53 showed efficacious effects of preventing recurrence or metastasis and improving both PFS and OS after a radical surgery in patients with NSCLC, and a better safety profile. These patients are still followed up to investigate the long-term beneficial effects of rAd-p53.

\section{MATERIALS AND METHODS}

\section{Study designs}

This was a phase II, randomized and open-labelled study to investigate the beneficial effect of the surgical wound surface injection of rAd-p53 after a radical surgery for patients with resectable NSCLC. The study was approved by the Ethics Committee of Daping Hospital of Third Military Medical University and registered in www. ClinicalTrials.gov (NCT01574729).

\section{Eligibility}

All patients were histopathologically diagnosed with resectable stage I to IIA NSCLC, had Eastern Cooperative Oncology Group (ECOG) performance status of 0 or 1 , were with normal hemogram, blood coagulation, liver and kidney function tests, and were over 18 years old. In addition, there were no surgery contraindications such as severe cardiac disease, uncontrolled diabetes mellitus. All the patients provided written informed consent.

\section{Treatment}

Patients were randomly assigned to receive either a radical surgery alone $(\mathrm{S})$ or a radical surgery plus surgical wound surface injection of rAd-p53 (SP). In order to completely remove the primary tumor lesions, a segmentectomy, lobectomy, or pneumonectomy and systematic mediastinal lymphadenectomy were performed according to the lesion size, location, and number of lesions. For SP group, after removing the primary tumor and performing complete hemostasis, $2 \times 10^{12}$ viral units of rAd-p53 diluted into $10-15 \mathrm{ml}$ of physiological saline containing 1:5000 epinephrine were evenly injected into the surgical wound surface by multiple injection points and multiple directions at one point. To avoid injection the medication into blood vessel, before injecting, pull back slightly on plunger to check for blood in syringe.

All participating patients were followed up for at least 3 years. In the first year after surgery, a chest $\mathrm{x}$-ray and computed tomography scan were performed every 3 months, every 4 months in the second year and then every 6 months after 2 years.

\section{Statistical analysis}

SAS 9.3 was used for analysis of data. The recurrence or metastasis (Rec/Met) rate was calculated and comparison by chi-square test was performed between the two treatment arms. The PFS was defined as the time from surgery to Rec/ Met, or death, and OS was defined as from surgery to death. The PFS and OS were estimated using Kaplan-Meier methods and compared between the two groups using Logrank test. The hazard ratio of PFS and OS (SP vs. S group) were estimated by Cox model with tumor stage and age as a covariate. All the safety data were summarized.

\section{CONFLICTS OF INTEREST}

The authors declare no conflicts of interest.

\section{REFERENCES}

1. Scagliotti GV, Fossati R, Torri V, Crinò L, Giaccone G, Silvano G, Martelli M, Clerici M, Cognetti F, Tonato M, and Adjuvant Lung Project Italy/European Organisation for Research Treatment of Cancer-Lung Cancer Cooperative Group Investigators. Randomized study of adjuvant chemotherapy for completely resected stage I, II, or IIIA nonsmall-cell Lung cancer. J Natl Cancer Inst. 2003; 95: 1453-61.

2. Wright G, Manser RL, Byrnes G, Hart D, Campbell DA. Surgery for non-small cell lung cancer: systematic review and meta-analysis of randomised controlled trials. Thorax. 2006; 61: 597-603.

3. Sugimura H, Yang P. Long-term survivorship in lung cancer: a review. Chest. 2006; 129: 1088-97.

4. al-Kattan K, Sepsas E, Fountain SW, Townsend ER. Disease recurrence after resection for stage I lung cancer. Eur J Cardiothorac Surg. 1997; 12: 380-4.

5. Yano T, Okamoto T, Fukuyama S, Maehara Y. Therapeutic strategy for postoperative recurrence in patients with non-small cell lung cancer. World J Clin Oncol. 2014; 5: 1048-54.

6. Seemann S, Maurici D, Olivier M, Caron de Fromentel C, Hainaut P. The tumor suppressor gene TP53: implications for cancer management and therapy. Crit Rev Clin Lab Sci. 2004; 41: 551-83.

7. Kirsch DG, Kastan MB. Tumor-suppressor p53: implications for tumor development and prognosis. J Clin Oncol. 1998; 16: 3158-68. 
8. Yu M, Chen W, Zhang J. p53 gene therapy for pulmonary metastasis tumor from hepatocellular carcinoma. Anticancer Drugs. 2010; 21: 882-4.

9. Gibbons DL, Byers LA, Kurie JM. Smoking, p53 mutation, and lung cancer. Mol Cancer Res. 2014; 12: 3-13.

10. Bossi G. Mutant p53 and sIL-1Ra. Aging (Albany NY). 2015; 7:742-43. https://doi.org/10.18632/aging.100825.

11. Pan JJ, Zhang SW, Chen CB, Xiao SW, Sun Y, Liu CQ, Su X, Li DM, Xu G, Xu B, Lu YY. Effect of recombinant adenovirus-p53 combined with radiotherapy on long-term prognosis of advanced nasopharyngeal carcinoma. J Clin Oncol. 2009; 27: 799-804.

12. Liu S, Chen P, Hu M, Tao Y, Chen L, Liu H, Wang J, Luo J, Gao G. Randomized, controlled phase II study of post-surgery radiotherapy combined with recombinant adenoviral human p53 gene therapy in treatment of oral cancer. Cancer Gene Ther. 2013; 20: 375-8.

13. Non-small Cell Lung Cancer Collaborative Group. Chemotherapy in non-small cell lung cancer: a metaanalysis using updated data on individual patients from 52 randomised clinical trials. BMJ. 1995; 311:899-909.

14. Yang H, Yao F, Zhao Y, Zhao H. Clinical outcomes of surgery after induction treatment in patients with pathologically proven N2-positive stage III non-small cell lung cancer. J Thorac Dis. 2015; 7: 1616-23.

15. Waller D, Peake MD, Stephens RJ, Gower NH, Milroy R, Parmar MK, Rudd RM, Spiro SG. Chemotherapy for patients with non-small cell lung cancer: the surgical setting of the Big Lung Trial. Eur J Cardiothorac Surg. 2004; 26: 173-82.

16. Arriagada R, Bergman B, Dunant A, Le Chevalier T, Pignon JP, Vansteenkiste J, and International Adjuvant Lung Cancer Trial Collaborative Group. Cisplatin-based adjuvant chemotherapy in patients with completely resected nonsmall-cell lung cancer. N Engl J Med. 2004; 350: 351-60.

17. Scagliotti GV, Pastorino U, Vansteenkiste JF, Spaggiari L, Facciolo F, Orlowski TM, Maiorino L, Hetzel M, Leschinger M, Visseren-Grul C, Torri V. Randomized phase III study of surgery alone or surgery plus preoperative cisplatin and gemcitabine in stages IB to IIIA non-small-cell lung cancer. J Clin Oncol. 2012; 30: 172-8.

18. Suehisa H, Toyooka S. Adjuvant chemotherapy for completely resected non-small-cell lung cancer. Acta Med Okayama. 2009; 63: 223-30. 